\title{
A Study of EEG Alpha Wave Patterns in Patients with Cirrhosis and Minimal Hepatic Encephalopathy
}

\author{
Abhijit Swami ${ }^{*}$, Bhaskar Kanti Nath ${ }^{2}$, Riturag Thakuria ${ }^{2}$, Tanuk K Biswas ${ }^{2}$ and Tanushree \\ Debgupta $^{3}$ \\ ${ }^{1}$ Associate Professor of Medicine, Silchar Medical College, Assam, India \\ ${ }^{2}$ Assistant Professor of Medicine, Silchar Medical College, Assam, India \\ ${ }^{3}$ Resident Physician of Medicine, Silchar Medical College, Assam, India
}

Received: December 29, 2017; Accepted: January 31, 2018; Published: February 20, 2018

*Corresponding author: Abhijit Swami, Associate Professor of Medicine, Silchar Medical College, Assam, India, Email: drabhijitswami@gmail. com

\begin{abstract}
Background: Minimal Hepatic Encephalopathy (MHE) is now increasingly described in patients with cirrhosis without any overt abnormalities on clinical examination. The diagnosis of MHE is based on multiple tests including a combination of paper and pencil tests, computerised tests and neurophysiological tests.
\end{abstract}

Aim of the study: To study the alpha wave patterns in patients with MHE as this has been sparsely reported in the literature.

Methods: The study included 70 diagnosed cases of cirrhosis and an equal number of age and sex matched control who were subjected to two paper and pencil tests - NCT-A and DST along with EEG.

Results and observations: Of the 70 patients included in the study, 56 (80\%) was in CPC-A class. MHE was present in 52 (74.28\%) cases of which 38 belonged to CPC-A class. In patients with MHE, alpha wave frequency $(10.82 \pm 0.41 \mathrm{~Hz})$ was significantly lower than those in controls $(11.52 \pm 0.64 \mathrm{~Hz})(p<0.05)$. Alpha wave amplitude was lower in MHE patients $(35 \pm 2.78 \mu \mathrm{V})$ than in controls $(48.18 \pm 3.59 \mu \mathrm{V})(p<$ 0.05 ). The frequency and amplitude decreased with higher grades of CPC and higher NCT-A. No difference was noted in the pattern of other wave forms in EEG between patients with MHE and controls.

Conclusion: A lower frequency and amplitude of alpha wave along with abnormal NCT-A can be predictive of MHE in cirrhosis.

\section{Introduction}

Hepatic encephalopathty (HE) produces a wide spectrum of neurological and psychiatric manifestations ranging from subclinical alterations to coma of varying severity with profound influence on the quality of life and treatment decisions [1]. For assessment of severity of HE, West Haven's criteria and Glasgow coma scale are most widely used. The International Society for Hepatic Encephalopathy and Nitrogen Metabolism (ISHEN) has suggested that severity assessment of hepatic encephalopathy in patients with cirrhosis, whether made clinically or more objectively, should be continuous rather than categorical [2] and the term Covert Hepatic encephalopathy was coined which included Minimal Hepatic Encephalopathy (MHE) and Grade 1 West- Haven criteria.
The concept of Minimal hepatic encephalopathy (MHE) was developed in the 1970s as investigators found subtle disturbance in cerebral function in patient with cirrhosis who did not have any overt neurological dysfunction on clinical examination or in EEG but had abnormalities in simple neuropsychological tests. Minimal hepatic encephalopathy has been described in increasing number of cirrhotic patients, with incidence reported as high as $53-62 \%$ cirrhotic patients when tested by psychometric tests and neurophysiological tests [3]. A diagnosis of MHE has some implications on the activity profile of the patient, including ability to drive and perform complex functions.

There are several methods of diagnosing MHE including paper and pencil tests, computerised tests and neurophysiological tests. Paper and pencil tests include Psychometric Hepatic Encephalopathy Score (PHES) and Repeatable Battery for the Assessment of Neuropsychological Status (RBANS). The existing paper-pencil tests include line tracing test (LTT), serial dotting test (SDT), digit symbol test (DST) and number connection test A and B (NCT A and B). This battery measures psychomotor speed and precision, visual perception, visuo-spatial orientation, visual construction, concentration, attention and memory are simple to perform and can be completed in less than 20 minutes [4]. These tests are however limited by their inconsistencies in diagnosis of MHE due to lack of consensus regarding diagnostic criteria, increased reliability on motor functions, increased time required in conducting the tests, limitation of application due to poor educational qualifications of the patients and lack of reproducibility. Moreover, these tests assess only few discrete domains of impairment including attention span and some psychomotor skills [5].

Computerised tests include the Inhibitory Control Test (ICT), The Cognitive Drug Research (CDR), Scan Test and the STROOP App test. The Neurophysiological tests include Critical flicker frequency (CFF), EEG and Evoked Potentials which include Visual, auditory and somatosensory-evoked potentials. These tests require expensive equipments with highly trained personals for conducting and interpreting the results and in some cases 
require copyright for use of the software which may not be readily available. Although there is international consensus that psychometric tests are the gold standard in the diagnosis of MHE, no agreement exists as to what combination of tests should be carried out, and what the threshold value is at which MHE may be reliably diagnosed [6]. For diagnosis of MHE, at least two of the current validated testing strategies are required: which includes paper-pencil (PHES) and one of the following: computerized (CRT, ICT, SCAN, or Stroop) or neurophysiological (CFF or EEG) [3].

EEG variations have been described in MHE but these have been described as non specific. We postulated that since alpha waves have been linked to congnitive function, changes in alpha waves can be expected in patients with MHE in surface EEG.

\section{Methods}

\section{Study protocol}

The study involved patients diagnosed with cirrhosis attending Medicine Department of Silchar Medical College over a period of one year July 2015 to June 2016. The patients were investigated both on as outpatient and inpatient basis. 70 patients were included in the study group after obtaining informed consent. 70 age and sex matched healthy volunteers were recruited as controls with academic qualifications comparable to the study group. Diagnosis of cirrhosis was established by using non invasive tests which included APRI (AST/Platelet ratio index) $=$ AST $/$ upper limit of normal AST $*\left[100 /\right.$ Platelet count $\left(* 10^{3} /\right.$ $\mathrm{mm}^{3}$ ), radiologic imaging, and/or endoscopic features of portal hypertension. Child-Pugh Score was used to assess severity of cirrhosis and the patients were classified as CPC-A - C in cirrhotic cases without overt HE.

\section{Materials Recorded}

Detailed history and physical examination was performed in every case. Laboratory tests done in the patients include Haematological investigations like $\mathrm{Hb} \%$, ESR, TLC, DLC, Platelet count, MCV, MCH, Prothrombin time \& INR ; biochemical investigations like RPG/FPG; serum for: total protein and fraction, total bilirubin \& fraction, ALT, AST, AST/ALT ratio, electrolytes (sodium, potassium) urea and creatinine. Serological tests were done to diagnosed associated Hepatitis B and C infection. Patients who had ascites had their ascites fluid analysed for protein, cytological tests and SAAG (Serum Ascites Albumin Gradient). Other biochemical investigations were done as per assessment. Upper Gastrointestinal (UGI) Endoscopy, X-ray chest, Bone Marrow aspiration study and CT scan of Abdomen were done if required.

\section{Inclusion Criteria}

Any Cirrhotic patients without clinical signs of overt encephalopathy or past history of encephalopathy, with normal mental status on clinical examination and with normal motor and visual function.

\section{Exclusion Criteria}

Patients who had active variceal bleeding, bacterial infection (spontaneous bacterial peritonitis, sepsis or any other severe infection), renal insufficiency, active alcohol abuse, history of Overt Hepatic Encephalopathy and Grade I Hepatic Encephalopathy in West Haven criteria, electrolyte imbalance, consumption of lactulose, psychoactive drugs or any antibiotics during the past 2 weeks, history of epilepsy or seizures, presence of significant comorbidity, such as heart, respiratory, or renal failure, presence of hepatocellular carcinoma or other malignancy, previous TIPS or shunt surgery, patients on drugs like benzodiazepines, antidepressants, anticonvulsants, psychostimulative drugs and cognitive impairment attributed to other causes like stroke, Parkinson's disease, Alzheimers disease

An equal number of healthy people $(n=70)$ without any of the conditions in the exclusion criteria was taken as control for EEG analysis. Diagnostic Criteria for MHE was made in accordance to the guidelines developed by the Indian National Association for Study of the Liver (INASL) [7] which recommended at least two of the following tests- NCT-A or Finger counting Test - A (FCT-A), NCT-B or FCT-B, block design test and digit symbol test. In the present study, NCT-A and Digit Symbol Test (DST) was used to diagnose MHE. These tests were also done in the control group to determine the range. For patients who had discordant NCT-A and DST test results, a variation of $<10 \%$ between the tests was considered acceptable.

Number Connection Test-A (NCT-A) - The person or the subject was asked to take a demonstration test after proper explanation before the test proper. In the demonstration test, the subject was asked to connect circles number from 1 to 10 and randomly spread on the sheet with a pencil as quickly as possible. This was done to ensure correct a understanding about the procedure and then the test proper was carried on, where the subject was shown a sheet of paper with 25 numbered circles randomly spread over the paper and asked to connect the circles from 1 to 25 in consecutive with order pencil as quick as possible. Errors were not enumerated but were point to the subject who was asked to correct them. Test result was the needed by the subject in seconds including error correction time, as measured by a stop watch. A low score indicated a good performance.

Digit Symbol test (DST) - the subject was given a series of double-boxes with a number given in the upper part. The task was to draw a symbol pertinent to this number into the lower part of the boxes. Nine fixed pairs of numbers and symbols were given at the top of the test sheet. Test result was the number of boxes correctly filled within 90 seconds.

The time taken by the control group was $<240$ sec for NCT-A and $<90$ sec for DST. Values above this range were considered abnormal in the study group indicative of MHE.

MMSE (Mini mental state examination) was done in all cases to rule out other causes of dementia.

EEG - EEG analysis was done in awake subjects with both eyes open and eyes closed states, at least $3 \mathrm{~min}$ in each state using digital EEG equipment (RMS EEG 24 Brain View Plus, Chandigarh) 
and the International 10-20 scalp electrode placement system approved by International Federation of Societies for EEG and Clinical Neurophysiology. The recording was done for at least 30 minutes.

The MMSE, NCT-A and DST and EEG were preferably done on the same day and it was ensured that during EEG recording the subject was not hungry, anxious or sleepy.

\section{Results}

The study included 70 patients of which most of the patients were male. The mean age of the patients was $45.38 \pm 12.1$ years. The commonest cause of cirrhosis was alcohol related $-72.85 \%$ and majority of the patients were in Child-Pugh criteria a (80\%). Fatigue and anorexia were the commonest symptoms. Among the physical findings, edema and ascites were seen in $71.42 \%$ and $60 \%$ of patients respectively. Clinical jaundice was detected in $55.71 \%$ patients. Hepatomegaly and splenomegaly were seen in $22.85 \%$ and $15.71 \%$ patients in the study group (Table 1 ).

Table 1: Demographic, Clinical and Biochemical profile of Study population

\begin{tabular}{|c|c|}
\hline Characteristics & Data \\
\hline Age (years) & $45.38 \pm 12.1$ \\
\hline $\operatorname{Sex}(M / F)$ & $63 / 7$ \\
\hline Symptomatology & Number of cases (\%) \\
\hline Fatigue & $49(70)$ \\
\hline Anorexia & $43(61.42)$ \\
\hline Yellowish discoloration of urine/ eyes. & $39(55.71)$ \\
\hline Constipation & $31(44.28)$ \\
\hline Edema & $50(71.42)$ \\
\hline Icterus & $45(64.28)$ \\
\hline Pallor & $42(60)$ \\
\hline Ascites & $42(60)$ \\
\hline AST (IU/L) & $57.25 \pm 25.64$ \\
\hline ALT (IU/L) & $57.59 \pm 27.28$ \\
\hline INR & $1.38 \pm 0.238$ \\
\hline PT (seconds) & $13.84 \pm 0.77$ \\
\hline Serum Creatinine (mg/dl) & $0.92 \pm 0.17$ \\
\hline Serum Urea $(\mathrm{mg} / \mathrm{dl})$ & $20.57 \pm 5.02$ \\
\hline Serum Sodium (mmol/L) & $138.80 \pm 2.82$ \\
\hline $\mathrm{RBS}(\mathrm{mg} / \mathrm{dl})$ & $105.75 \pm 8.0$ \\
\hline \multicolumn{2}{|l|}{ Cause of Cirrhosis } \\
\hline Alcohol related & $51(72.85 \%)$ \\
\hline HBV related & $5(7.14 \%)$ \\
\hline HCV related & $1(1.42 \%)$ \\
\hline Other & $13(18.57 \%)$ \\
\hline
\end{tabular}

In the study, $80 \%$ patients were in CPC-A class. Alcohol related cirrhosis was the commonest etiologic group, seen in $72.85 \%$ cases. In $18.57 \%$ cases, the cause of cirrhosis could not be determined.

MHE was diagnosed in $52(74.28 \%)$ based on NCT-A test result of $>240 \mathrm{sec}$ and DST $>90 \mathrm{sec} .36$ (69.23\%) patients with MHE required between $241-480 \mathrm{sec}$ to do NCT-A. The time taken to do NCT-A was more in patients with CPC-C who required $>450$ seconds or more but there was only 2 ( $2.85 \%$ ) patients in the group (Table 2).

\begin{tabular}{|c|c|c|c|}
\hline & Cases $(n=52)$ & \multicolumn{2}{|c|}{ Controls $(n=70)$} \\
\hline Age & $45.38 \pm 12.1$ & \multicolumn{2}{|c|}{$46.42 \pm 14.5$} \\
\hline $\operatorname{Sex}(M / F)$ & $63 / 7$ & \multicolumn{2}{|c|}{$62 / 8$} \\
\hline Alpha frequency (Hz) & $10.82 \pm 0.41$ & $11.52 \pm 0.64$ & $P<0.05$ \\
\hline Alpha Amplitude $(\mu \mathrm{V})$ & $35.54 \pm 2.78$ & $48.18 \pm 3.59$ & $P<0.05$ \\
\hline alpha amplitude $<30 \mu \mathrm{V}$ & 24 & 2 & \\
\hline $\begin{array}{l}\text { Alpha wave frequency } \\
\qquad(8-9 \mathrm{~Hz})\end{array}$ & 15 & 1 & \\
\hline
\end{tabular}

EEG findings in the patients with MHE and equal number of controls showed slower frequency of alpha waves in patients with MHE $(10.82 \pm 0.41 \mathrm{~Hz}$ vs. $11.52 \pm 0.64 \mathrm{~Hz} ; p<0.05) .15 / 52$ (28.84\%) of MHE patients had alpha waves of frequency 8-9 Hz. Only $1(.42 \%)$ among the healthy controls had alpha wave frequency in this range.

Patients with MHE had lower amplitude of alpha waves than control (35.54 $\pm 2.78 \mu \mathrm{V} ; P<0.05$ ). 24 patients with MHE (46.15\%) had alpha wave amplitude of less than $30 \mu \mathrm{V}$ which was seen in $2(2.85 \%)$ of the control population.

Both the groups did not have any difference in alpha wave reactivity to eye opening. There was no difference in the amplitude of the alpha waves between right and left brain hemispheres in both the groups and in distribution of the waves (Table 3).

$80 \%$ of the patients with cirrhosis were in CPC-A class. All 18 of the 70 patients with cirrhosis and without MHE (NCT-A $\leq$ $240 \mathrm{sec}$ ) belonged to CPC-A class . There were only 2 patients in CPC-C class and all had NCT-A > $481 \mathrm{sec}$. Alpha wave frequency and amplitude was found to be lower in patients with increased NCT-A time. Patients in CPC-A class with MHE had alpha wave frequency $>10 \mathrm{~Hz}$ which was lower than in patients without MHE. Alpha amplitude $<40 \mu \mathrm{V}$ was seen in all patients with MHE with lower amplitudes noted in CPC - B/C classes (Table 4).

Patients with MHE had slower beta wave frequency $(15.35 \mathrm{~Hz}$ vs $16.67 \mathrm{~Hz} ; p>0.05)$ and lower amplitude ( $9.02 \mathrm{mV}$ vs. $9.41 \mathrm{mV}$; $p>0.05$ ) though the findings were not statistically significant. There was no difference in the amplitude and frequency of theta and delta waves among patients with MHE and the control group. 
Table 3: Alpha wave pattern according to CPC and NCT-A values

\begin{tabular}{|c|c|c|c|c|c|}
\hline \multirow{2}{*}{ Child-Pugh Class(CPC) } & \multirow{2}{*}{$\%$ of patients } & \multicolumn{2}{|c|}{ NCT - A } & \multicolumn{2}{|c|}{ EEG alpha wave (Mean \pm SD) } \\
\hline & & Time (sec) & Number & Freq $(\mathrm{Hz})$ & Voltage $\mu V$ \\
\hline \multirow{3}{*}{ CPC-A } & \multirow{3}{*}{$56(80 \%)$} & $\leq 240 \mathrm{sec}$ & 18 & $10.98 \pm 0.4$ & $44.56 \pm 3.64$ \\
\hline & & $241-480$ & 36 & $10.22 \pm 0.16$ & $36.45 \pm 2.88$ \\
\hline & & $\geq 481$ & 2 & 10.12 & 33.86 \\
\hline \multirow{3}{*}{ CPC-B } & \multirow{3}{*}{$12(17.14 \%)$} & $\leq 240 \mathrm{sec}$ & 0 & - & - \\
\hline & & $241-480$ & 8 & $9.96 \pm 0.16$ & $37.64 \pm 2.6$ \\
\hline & & $\geq 481$ & 4 & $9.43 \pm 0.44$ & $31 \pm 0.3$ \\
\hline \multirow{3}{*}{$\mathrm{CPC}-\mathrm{B}$} & \multirow{3}{*}{$2(2.85 \%)$} & $\leq 240 \mathrm{sec}$ & 0 & - & - \\
\hline & & $241-480$ & 0 & - & - \\
\hline & & $\geq 481$ & 2 & 8.22 & 29 \\
\hline
\end{tabular}

Table 4: Other EEG wave pattern

\begin{tabular}{|c|c|c|c|c|}
\hline \multicolumn{2}{|c|}{ Other wave forms } & \multirow{2}{*}{$\begin{array}{c}\text { Cases } \mathbf{n}=\mathbf{5 2} \\
15.35\end{array}$} & \multirow{2}{*}{$\begin{array}{c}\text { Controls } \mathbf{n}=\mathbf{7 0} \\
16.67\end{array}$} & \multirow{2}{*}{$\begin{array}{l}\text { p value } \\
P>0.05\end{array}$} \\
\hline & Frequency (Hz) (mean value) & & & \\
\hline Beta Activity & Amplitude (mV) (mean value) & 9.02 & 9.41 & $P>0.05$ \\
\hline \multirow{2}{*}{ Theta Activity } & Frequency (Hz) (mean value) & 5.74 & 6.31 & $P>0.05$ \\
\hline & Amplitude (mV) (mean value) & 10.22 & 10.04 & $P>0.05$ \\
\hline \multirow{2}{*}{ Delta Activity } & Frequency (Hz) (mean value) & 3.12 & 2.45 & $P>0.05$ \\
\hline & Amplitude (mV) (mean value) & 53.81 & 53.31 & $P>0.05$ \\
\hline
\end{tabular}

\section{Discussion}

In the present study, males outnumbered females (M/F$63 / 7)$ with the average age at presentation being $45.38 \pm 12.1$ years. This findings were similar to the results obtained in studies by Sharma et al who found the presenting age to be $44.0 \pm 11.4$ years with higher incidence in males (M:F:75:16)[8]. The errors on NCT-A tend to increase with age [9], but as the mean age of the patients was $45.38 \pm 12.1$ years in this study, this was not considered to significantly influence the results.

Alcoholism was the commonest cause of cirrhosis 51 (72.85\%), with incidence of chronic hepatitis B being 5(7.14\%). 13 patients had cirrhosis of unknown etiology. This finding was different from that of Maric et al [10] where $43 \%$ had chronic hepatitis B infection and 50\% had chronic hepatitis C viral infection as well as from the study of Sharma et al 8 where chronic Hepatitis B infection was the commonest type. Wang et al in their study found chronic hepatitis B infection and concomitant alcoholic liver disease as the second most common cause of MHE [11].

Most of the patients had multiple presenting complaints with fatigue, anorexia and constipation being the commonest. Physical examination revealed pedal edema (71.42\%), jaundice (64.28\%) and ascites (60\%) as the commonest findings. Hepatosplenomegaly was present in $22.85 \%$ and $15.71 \%$ of cases.

In the present study, based on the NCT-A and DST, 52 patients (74.28\%) were diagnosed to have MHE. This figure was higher than in studies from China $[12,13]$ and another study from India [14] where the incidence of MHE among cirrhotic patients varied from $48 \%-49.1 \%$. That there was a demographic variation was evident from a Korean study where the incidence of MHE was found to be $25.6 \%$ [15].

One possible explanation about the difference could be that the liver function of patients in the studies was different. This was evident from the findings that $80 \%$ of patients in the present study were in CPC-A which correlated with that of the Korean study $(80.6 \%)$ whereas the proportion of Child A in the Chinese study ${ }^{12}$ and the Indian study [14] were $45.3 \%$ and $22.0 \%$, respectively.

Patients with CPC-A had lower NCT-A (120 - $264 \mathrm{sec}$ - mean value $188 \pm 36 \mathrm{sec}$ )then compared to those in CPC-B and CPC-A who had a higher NCT-A. 16 (22.85\%) CPC-C patients had NCT-A exceeding $450 \mathrm{sec}$ which was not seen in the control population. Better psychometric test performance was seen with lower CPC grading in other studies as well [10]. CPC score was found to have a positive predictive value in patients developing MHE and this finding was similar to other studies [3]. Groweneweg et al found MHE incidence of $15 \%$ in patients with normal liver function (CPC-A) while MHE was present in half of the patients with advanced cirrhosis -CPC-B/C [16]

The EEG is a reliable testing procedure for several brain dysfunctions. A major advantage of utilisation of EEG in brain dysfunction is its independence from age, education and cultural effects.

EEG changes were first described in Hepatic encephalopathy (HE) in 1950s, when Foley, Watson et al described the monomorphic 2 per second waves in the frontal region of patients with clinically overt HE17. Parsons- Smith described EEG changes 
in HE which comprised of 5 groups (A to E) and showed a fair correlation with the evolution of the disease condition [18]. - The initial changes being generalised suppression of alpha waves and its replacement by beta waves.

In contrast to HE, MHE has not been associated with typical EEG changes as is described in overt hepatic encephalopathy. However, as the psychometric tests demonstrate subtle alterations in higher mental functions occur in MHE, it is probably logical to expect some EEG changes in MHE. Using spectral analysis Amodio and co-workers observed pathological slowing of the EEG in 31 of 100 cirrhotic patients without clinical signs of HE [19]. Montagnese et al observed alterations of the EEG considering the mean dominant frequency in only $8.5 \%$ of cirrhotic patients with MHE [20]. However, the sensitivity of the EEG for lower grades of HE is limited and thus its use for diagnosing minimal HE is controversial.

Alpha wave was the first EEG pattern to be described and it continues to be the most commonly noted rhythm in clinical EEG interpretation because it is reproducible and easily recognised. Alpha rhythm may have a frequency of between 8 and $13 \mathrm{~Hz}$, but in most adults it is between 9 and $11 \mathrm{~Hz}$. The alpha wave amplitude varies among individuals and usually is between 40 and $50 \mu \mathrm{V}$ in adults. Occipital Alpha is the dominant rhythm with a frequency of $8-13 \mathrm{~Hz}$ in relaxed wakefulness in $85 \%$ of healthy adults with closed eyes and generally attenuates (diminishes) with eye opening, mental activity, and drowsiness. The alpha generator is thought to be located within the thalamus and regions of occipital and parieto-occipital cortex [21].

Alpha waves are a good indicator of cognitive function and alpha wave of $>10 \mathrm{~Hz}$ is an important measure of cognitive and memory performance $[22,23]$. The normal frequency of alpha waves may decrease in certain neurological disorders like dementia, medication effects and age related cognitive decline. Alpha waves are the only brain waves which are influenced by tasks and / or task demands [22].

Alpha wave variations are known to happen in response to stimulus and/or tasks and one of the implicit assumptions in EEG research is that alpha waves have an impact on information processing [23]. As MHE is considered a subclinical stage of HE, at least theoretically the EEG abnormalities should correlate with the paper and pencil tests which was to an extent validated in our study. In our study, the amplitudes and frequency of alpha waves were within the normal range but patients with MHE had values on the lower side of normal.

Patients in CPC-A had higher alpha frequency and amplitude than as compared to patients in CPC-B. This change in alpha waves has been found to correlate with the increasing severity of liver disease. The study found agreement between results of NCT-A and alpha wave changes in the EEG with decreasing alpha wave amplitude and lower frequency noted in patients with higher grades of CPC. As the study group had patients with mean age < 50 years, the slowing of alpha waves which occur with increasing age could be discounted and the slowing could be attributed to MHE - a finding which correlated with NCT-A findings.
Our study confirmed the findings of earlier studies of increased abnormalities in psychometric tests with higher grades of liver dysfunction in cirrhotic patients. We found that a majority (75\%) of patients with MHE had alpha wave changes as compared to normal controls which included decrease in amplitude and lowered frequency - findings which were statistically significant when compared to normal controls. This finding was higher than those of Quero et al who found 17\% EEG abnormalities in their study on MHE in cirrhotic patients [24].

\section{Limitation of the study}

A perceived limitation of the study is the smaller number of patients. The study also did not follow up the patients after initial diagnosis of MHE.

\section{Conclusion}

The study found a high incidence of MHE among cirrhotic patients. Patients with lower grades of liver dysfunction (CPC-A) had lower NCT-A. Alpha wave frequency and voltage was found to be higher in patients with CPC-A than in patients with in CPCB/C. Patients with MHE had alpha waves of lower amplitudes $(35 \pm 2.78 \mu \mathrm{V})$ and frequency $(10.82 \pm 0.41 \mathrm{~Hz})$ than the control population. Alpha wave frequency and voltage was found to be higher in patients with CPC-A than in patients with in CPC$B / C$. These findings suggest that EEG alpha wave morphology - frequency $<10 \mathrm{~Hz}$ and amplitude $<40 \mu \mathrm{V}$ can be used as a potential supplement to other diagnostic tests of MHE as EEG is readily available, reproducible and is not prone to be affected by the educational qualification of the patient.

\section{Acknowledgement}

Departmental staff of Medicine.

\section{Ethical approval}

The study design was approved by the ethics committee of the College

\section{References}

1. Vilstrup H, Amodio P, Bajaj J, Cordoba J, Ferenci P, Mullen KD, et al. AASLD PRACTICE GUIDELINE, Hepatic Encephalopathy in Chronic Liver Disease: 2014 Practice Guideline by the American Association for the Study of Liver Diseases and the European Association for the Study of the Liver. Journal of Hepatology. 2014;61(3). DOI 10.1002/ hep. 27210

2. Bajaj JS, Cordoba J, Mullen KD, Amodio P, Shawcross DL, Butterworth $\mathrm{RF}$, et al. Review article: The design of clinical trials in hepatic encephalopathy - An International Society for Hepatic Encephalopathy and Nitrogen Metabolism (ISHEN) consensus statement. Aliment Pharmacol Ther. 2011;33(7):739-747. doi: 10.1111/j.13652036.2011.04590.x

3. Das A, Dhiman RK, Saraswat VA, Verma M, Naik SR. Prevalence and natural history of subclinical hepatic encephalopathy in cirrhosis. J Gastroenterol Hepatol. 2001;16(5):531-535.

4. Sharma P, Sharma BC, Puri V, Sarin SK. Critical flicker frequency: diagnostic tool for minimal hepatic encephalopathy. J Hepatol. 2007;47(1):67-73.

5. Weissenborn K, Ennen JC, Schomerus H, Rückert N, Hecker H. 
Neuropsychological characterization of hepatic encephalopathy. ] Hepatol. 2001;34(5):768-773.

6. Iduru S, Hisamuddin Kand Mullen KD. Minimal hepatic encephalopathy: simplifying its diagnosis. Am J Gastroenterol. 2007;102(7):1537-1538.

7. Tianzuo Zhan and Wolfgang Stremmel. The Diagnosis and Treatment of Minimal Hepatic Encephalopathy. Dtsch Arztebl Int. 2012;109(10):180187. doi: $10.3238 /$ arztebl.2012.0180

8. Dhiman RK, Saraswat VA, Sharma BK, Sarin SK, Chawla YK, Butterworth $\mathrm{R}$, et al. Minimal hepatic encephalopathy: Consensus statement of a working party of the Indian National Association for Study of the Liver J Gastroenterol Hepatol. 2010;25(6):1029-41. doi: 10.1111/j.14401746.2010.06318.x

9. Sharma P and Kumar A. Minimal Hepatic Encephalopathy in Patients with Cirrhosis by Measuring Liver Stiffness and Hepatic Venous Pressure Gradient. Saudi J Gastroenterol. 2012;18(5):316-321.

10.Jae Yoon Jeong, Dae Won Jun, Daiseg Bai, Ji Yean Kim, Joo Hyun Sohn, Sang Bong Ahn, et al. Validation of a Paper and Pencil Test Battery for the Diagnosis of Minimal Hepatic Encephalopathy in Korea. J Korean Med Sci. 2017;32(9):1484-1490. doi: 10.3346/jkms.2017.32.9.1484

11.Marić D, Klasnja B, Filipović D, Brkić S, Ružić M and Bugarski V. Minimal hepatic encephalopathy in patients with decompensated liver cirrhosis. Acta Clin Croat. 2011;50(3):375-380.

12.ji-Yao Wang, Ning-Ping Zhang, Bao-Rong Chi, Yu-Qing Mi, Li-Na Meng, Ying-Di Liu, et al. Prevalence of minimal hepatic encephalopathy and quality of life evaluations in hospitalized cirrhotic patients in China. World J Gastroenterol. 2013;19(30):4984-4991. doi: 10.3748/wjg v19.i30.4984

13.Su-Wen Li, Kai Wang, Yong-Qiang Yu, Hai-Bao Wang, Yuan-Hai Li, and Jian-Ming Xu. Psychometric hepatic encephalopathy scores for diagnosis of minimal hepatic encephalopathy in China. World J Gastroenterol. 2013;19(46):8745-8751. doi: 10.3748/wjg.v19. i 46.8745

14.Zeng Z, Li YY, Nie YQ. An epidemiological survey of subclinical hepatic encephalopathy. Zhonghua Gan Zang Bing Za Zhi. 2003;11(11):680682.

15.Dhiman RK, Kurmi R, Thumburu KK, Venkataramarao SH, Agarwal
R, Duseja A, et al. Diagnosis and prognostic significance of minimal hepatic encephalopathy in patients with cirrhosis of liver. Dig Dis Sci. 2010;55(8):2381-2390. doi: 10.1007/s10620-010-1249-7

16.Seo YS, Yim SY, Jung JY, Kim CH, Kim JD, Keum B, et al. Psychometric hepatic encephalopathy score for the detection of minimal hepatic encephalopathy in Korean patients with liver cirrhosis. J Gastroenterol Hepatol. 2012;27(11):1695-1704. doi: 10.1111/j.14401746.2012.07217.x

17.Groeneweg M, Moerland W, Quero JC, Hop WC, Krabbe PF, Schalm SW. Screening of subclinical hepatic encephalopathy. J Hepatol. 2000;32(5):748-753.

18.FOLEY JM, WATSON CW, ADAMS RD. Significance of electroencephalographic changes in hepatic coma. Trans Am Neurol Assoc. 1950;51:161-165.

19.PARSONS-SMITH BG, SUMMERSKILL WH, DAWSON AM, SHERLOCK S. The electroencephalograph in liver disease. Lancet. 1957;273(7001):867-871.

20.Amodio P, Campagna F, Olianas S, Iannizzi P, Mapelli D, Penzo M, et al. Detection of minimal hepatic encephalopathy: normalization and optimization of the Psychometric Hepatic Encephalopathy Score. A neuropsychological and quantified EEG study. J Hepatol. 2008;49(3):346-353.

21. Montagnese S, Jackson C, Morgan MY. Spatio-temporal decomposition of the electroencephalogram in patients with cirrhosis. J Hepatol. 2007;46(3):447-458.

22.Goldman RI, Stern JM, Engel, J Jr, and Cohen MS. Simultaneous EEG and fMRI of the alpha rhythm Neuroreport. 2002;13(18):2487-2492.

23. Klimesch W. Alpha-band oscillations, attention, and controlled access to stored information. Trends Cogn Sci. 2012;16(12):606-617. doi: 10.1016/j.tics.2012.10.007

24.Knyazev GG, Savostyanov AN, Levin EA. Alpha synchronization and anxiety: Implications for inhibition vs. alertness hypotheses. 2006;59(2):151-158.

25.Quero JC, Hartmann IJ, Meulstee J, Hop WC, Schalm SW. The diagnosis of subclinical hepatic encephalopathy in patients with cirrhosis using neuropsychological tests and automated electroencephalogram analysis. Hepatology. 1996;24(3):556-560. 